Chemical Shift MR Imaging Methods for the Quantification

of Transcatheter Lipiodol Delivery to the Liver: Preclinical Feasibility Studies in a Rodent Model ${ }^{1}$

Xiaoming Yin, PhD

Yang Guo, MD

Weiguo Li, PhD

Eugene Huo, MD

Zhuoli Zhang, MD, PhD

Jodi Nicolai, MS

Robert A. Kleps, PhD

Diego Hernando, PhD

Aggelos K. Katsaggelos, PhD

Reed A. Omary, MD, MS

Andrew C. Larson, PhD
Purpose:

Materials and Methods:

Results:

Conclusion:

${ }^{1}$ From the Department of Electrical Engineering and Computer Science (X.Y., A.K.K., A.C.L.) and Department of Biomedical Engineering (J.N., R.A.O., A.C.L.), Northwestern University, Evanston, III; Department of Radiology, Northwestern University, 737 N Michigan Ave, Suite 1600, Chicago, IL 60611 (X.Y., Y.G., W.L., E.H., Z.Z., J.N., R.A.O., A.C.L.); Robert H. Lurie Comprehensive Cancer Center, Northwestern University, Chicago, III (Z.Z., R.A.O., A.C.L.); Research Resources Center (R.A.K.) and Department of Bioengineering (A.C.L.), University of Illinois at Chicago, Chicago, III; and Department of Radiology, University of Wisconsin-Madison, Madison, Wis (D.H.). Received September 7, 2011; revision requested October 21; revision received December 2; accepted December 28; final version accepted January 18, 2012. Address correspondence to A.C.L. (e-mail: a-larson@northwestern.edu).

o RSNA, 2012
To demonstrate the feasibility of using chemical shift magnetic resonance $(\mathrm{MR})$ imaging fat-water separation methods for quantitative estimation of transcatheter lipiodol delivery to liver tissues.

Studies were performed in accordance with institutional Animal Care and Use Committee guidelines. Proton nuclear MR spectroscopy was first performed to identify lipiodol spectral peaks and relative amplitudes. Next, phantoms were constructed with increasing lipiodol-water volume fractions. A multiecho chemical shift-based fatwater separation method was used to quantify lipiodol concentration within each phantom. Six rats served as controls; 18 rats underwent catheterization with digital subtraction angiography guidance for intraportal infusion of a $15 \%, 30 \%$, or $50 \%$ by volume lipiodol-saline mixture. MR imaging measurements were used to quantify lipiodol delivery to each rat liver. Lipiodol concentration maps were reconstructed by using both single-peak and multipeak chemical shift models. Intraclass and Spearman correlation coefficients were calculated for statistical comparison of MR imaging-based lipiodol concentration and volume measurements to reference standards (known lipiodol phantom compositions and the infused lipiodol dose during rat studies).

Both single-peak and multipeak measurements were well correlated to phantom lipiodol concentrations $\left(r^{2}>\right.$ 0.99). Lipiodol volume measurements were progressively and significantly higher when comparing between animals receiving different doses $(P<.05$ for each comparison). MR imaging-based lipiodol volume measurements strongly correlated with infused dose (intraclass correlation coefficients $>0.93, P<.001$ ) with both single- and multipeak approaches.

Chemical shift MR imaging fat-water separation methods can be used for quantitative measurements of lipiodol delivery to liver tissues.

${ }^{\circ}$ RSNA, 2012 
$\mathbf{T}$ ranscatheter arterial chemoembolization (TACE) is a widely accepted palliative therapy for the treatment of hepatocellular carcinoma (HCC) $(1,2)$. TACE involves selective placement of a catheter to deliver drugs and embolic materials directly into the vascular supply of targeted tumors. TACE has been proven to improve patient survival and reduce tumor burden $(3,4)$.

During TACE, a mixture of drugs emulsified within iodinated oil (lipiodol) is selectively infused to treat the targeted lesions. Lipiodol serves a pivotal role as both drug carrier and embolic agent (5). Lipiodol can be visualized intraprocedurally to confirm delivery with fluoroscopy (6) or postprocedurally with computed tomography (CT) $(7,8)$. CT performed after TACE provides an estimate of overall procedural efficacy to predict therapeutic outcome (9).

The distribution of the lipiodol drug emulsion can be qualitatively visualized with digital subtraction angiography (DSA) methods or with follow-up CT; however, quantitatively estimating the intrahepatic distribution of lipiodol after transcatheter infusion can be challenging. Recently, rotational DSA/Carm CT methods have been proposed for intraprocedural lipiodol distribution measurements (10) but these methods provide relatively limited spatial coverage and require exposure to a relatively high dose of ionizing radiation.

With the increasing availability of combined DSA/magnetic resonance (MR) imaging procedure suites $(11,12)$, it is now possible to use DSA to guide catheter placement and MR imaging as a complimentary modality allowing a broad range of additional quantitative imaging measurements (13). Prior studies performed in patients after undergoing TACE procedures have reported T1- and T2-weighted signal intensity changes attributed to lipiodol deposition (14); however, these signal

\section{Advance in Knowledge}

- Multiecho chemical shift-based MR imaging methods can be used to quantitatively assess lipiodol delivery to liver tissues. intensity changes were variable between patients and thus not a viable option for quantitative estimation of lipiodol delivery. Alternatively, the fatty acids within lipiodol should permit in vivo quantification of intrahepatic biodistribution with chemical shift-based fat-water separation techniques (15).

Fat-water imaging methods have rapidly evolved $(16,17)$ with strategies used to overcome the deleterious effect of field inhomogeneties (18). Recently developed spectral modeling approaches $(19,20)$, the so-called multipeak methods, can improve the accuracy of fat-water measurements. The feasibility of detecting lipiodol delivery with fat-water imaging techniques was recently demonstrated clinically in patients with HCC (21).

The purpose of our study was to demonstrate the ability to use chemical shift-based fat-water separation methods for quantitative estimation of lipiodol concentrations following transcatheter delivery to liver tissues.

\section{Materials and Methods}

\section{Nuclear MR Spectroscopy of Lipiodol}

Lipiodol (Guerbet, Bloomington, Ind), also previously marketed in the United States as ethiodol, is a mixture of ethyl esters of poppy seed oil to which iodine has been added. The iodine adds to the unsaturated bonds in the fatty acids to produce a mixture of iodostearates and stearate esters. Proton $\left({ }^{1} \mathrm{H}\right)$ nuclear MR spectroscopy measurements were performed to identify chemical structures in lipiodol and determine the position and relative amplitude of ${ }^{1} \mathrm{H}$ spectral peaks; these were later included in a multifrequency signal model during chemical shiftbased MR imaging measurements of lipiodol concentration (R.A.K., a physicist

\section{Implication for Patient Care}

- Accurate quantification of lipiodol distributions within the liver could permit early prediction of therapy response after catheterdirected infusion of this embolic drug delivery medium. with more than 20 years of experience in the field of nuclear MR spectroscopy).

Lipiodol dissolved in denatured chloroform was analyzed by means of a 360-MHz spectrometer (Bruker Avance; Bruker Medical, Ettlingen, Germany) by using ${ }^{1} \mathrm{H}$ and carbon $13\left({ }^{13} \mathrm{C}\right)$ techniques (single-pulse sequence, $30^{\circ}$ flip angle, and 15-second recycle delay interval to ensure that resulting peak areas were quantitative). Lipiodol is not a pure compound with the exact chemical structure unknown, but many signals in its ${ }^{1} \mathrm{H}$ spectrum can be assigned by using their chemical shifts. A verification of these assignments was performed with ${ }^{13} \mathrm{C}$ attached proton tests, two-dimensional ${ }^{1} \mathrm{H}^{-1} \mathrm{H}$ correlation and ${ }^{1} \mathrm{H}-{ }^{13} \mathrm{C}$ heteronuclear multiple-quantum correlation spectroscopy.

\section{Chemical Shift Lipiodol-Water Separation Methods}

A multiecho variable projection (VARPRO)-based fat-water separation

\section{Published onlin}

10.1148/radiol.12111916 Content code: GI

Radiology 2012; 263:714-722

\section{Abbreviations:}

DSA = digital subtraction angiography

HCC $=$ hepatocellular carcinoma

MGRE = multiple gradient-recalled echo

TACE = transcatheter arterial chemoembolization

VARPRO $=$ variable projection

\section{Author contributions:}

Guarantors of integrity of entire study, X.Y., A.C.L.; study concepts/study design or data acquisition or data analysis/ interpretation, all authors; manuscript drafting or manuscript revision for important intellectual content, all authors; approval of final version of submitted manuscript, all authors; literature research, X.Y., Y.G., W.L., E.H., Z.Z., A.K.K., R.A.O., A.C.L.; experimental studies, X.Y., Y.G., W.L., E.H., Z.Z., J.N., R.A.K., A.K.K., R.A.O., A.C.L.; statistical analysis, X.Y., Y.G., W.L., E.H., A.K.K., R.A.O.; and manuscript editing, X.Y., Y.G., W.L., E.H., Z.Z., R.A.K., D.H., A.K.K., R.A.O., A.C.L.

\section{Funding:}

This research was supported by the National Cancer Institute (grant CA134719) and the National Center for Research Resources (grant UL1RR0254741), National Institutes of Health, through the Clinical and Translational Science Awards Program, a trademark of DHHS, part of the Roadmap Initiative, "Re-Engineering the Clinical Research Enterprise."

Potential conflicts of interest are listed at the end of this article. 
method was used for the current study (22). These VARPRO-based methods involve jointly solving for both chemical shift signal components and field inhomogeneities; VARPRO permits the use of both single- and multipeak chemical shift signal models $(22,23)$.

Equations (1) and (2) model the signal intensity in a single voxel recorded during a multiple gradient-recalled echo (MGRE) readout with $t_{n}$ the echo formation time for the $n$th echo. For the single-peak signal model shown in Equation (1), the signal intensities for water and lipiodol components are represented by $s_{w}$ and $s_{L}$, respectively, with $f_{B}$ the local frequency offset due to the presence of an inhomogeneous static field. The single-peak resonance frequency for lipiodol at $1.5 \mathrm{~T}, f_{L}$, was determined to be $-216 \mathrm{~Hz}$ (principle peak at $\sim 1.3$ parts per million in Fig $1)$. For the multipeak model shown in Equation (2), the lipiodol signal was represented with $M$ equal to four peaks with the amplitudes of these respective peaks $\alpha_{m}(23,24)$ and corresponding resonant frequencies $f_{L, m}$ (see Results for specific amplitudes and frequencies):

$$
\begin{gathered}
S_{\left(t_{n}\right)}=S_{W} e^{j 2 p f_{B} t_{n}}+s_{L} e^{j 2 p\left(f_{L}+f_{B}\right) t_{n}} \\
S_{\left(t_{n}\right)}=S_{W} e^{j 2 \pi f_{B} t_{n}}+s_{L} e^{j 2 \pi\left(f_{L}+f_{B}\right) t_{n}} \\
S_{\left(t_{n}\right)}=e^{j 2 \pi f_{B} t_{n}}\left(S_{W}+s_{L} \sum_{m=1}^{M} \alpha_{m} e^{j 2 \pi\left(f_{L, m}\right) t_{n}}\right) \\
S_{\left(t_{n}\right)}=e^{j 2 \pi f_{B} t_{n}}\left(s_{W}+s_{L} \sum_{m=1}^{M} \alpha_{m} e^{j 2 \pi\left(f_{L, m}\right) t_{n}}\right)
\end{gathered}
$$

VARPRO-based fat-water separation methods consider a system of equations representing $S\left(t_{n}\right)$ for all voxels as a separable nonlinear least-squares fitting problem $(22,25)$. VARPRO-based methods provide robust maximumlikelihood estimates of $s_{w}, s_{L}$, and $f_{B}$ while imposing spatial smoothness as a priority for solution of the inhomogeneous static field $\left(f_{B}[\mathrm{x}, \mathrm{y}]\right)(22)$. These VARPRO-based fat-water separation methods involve first estimating field inhomogeneity $f_{B}$ within a constrained range of frequency values; $f_{B}$ is then used when simultaneously solving for fat and water signal components from the complex images. Specific VARPRO implementation details for the current studies included field inhomogeneity bounds from -800 to $800 \mathrm{~Hz}$ discretized into 600 field values, $5 \times 5$ window for Markov random field neighborhood, and regularization parameter $\mu=0.02$ (23).

\section{Lipiodol Phantom Preparation}

Agar phantoms were constructed with lipiodol-water volume fractions of 0 , $5 \%, 10 \%, 20 \%$, and $40 \%$ based on previously described oil/water phantom preparation methods $(26,27)$ (J.N., 3 years of experience). In brief, each $10 \mathrm{~mL}$ phantom included 43 $\mathrm{mmol} / \mathrm{L}$ sodium dodecyl sulfate and sodium chloride, $3.75 \mathrm{mmol} / \mathrm{L}$ sodium azide, $0.3 \mathrm{mmol} / \mathrm{L}$ gadopentetate dimeglumine, and $2 \% \mathrm{wt} / \mathrm{vol}$ agarose. The corresponding lipidol concentrations for these five phantoms were 0 , $0.064,0.10,0.256$, and $0.512 \mathrm{~g} / \mathrm{mL}$, respectively.

\section{Animal Model Preparation}

Studies were performed in accordance with our institutional Animal Care and Use Committee guidelines. Twenty-four adult Sprague-Dawley rats (Charles River Laboratories, Wilmington, Mass) were divided into four groups (six rats per group), with groups 1, 2, and 3 receiving transcatheter intrahepatic 1 $\mathrm{mL}$ infusions of $15 \%, 30 \%$, and $50 \%$ by volume lipiodol-saline mixture, respectively; group 4 served as a control without operation or lipiodol infusion. Rats were anesthetized with intramuscular injection of ketamine (75-100 mg per kilogram of body weight) and xylazine $(2-6 \mathrm{mg} / \mathrm{kg}$ ) (animal model studies were performed in concert by Y.G. [4 years experience], W.L. and H.G. [2 years experience], and Z.L. [more than 10 years experience]).

For rats in groups 1, 2, and 3, skin incisions and dissections were performed to expose the portal vein. Sutures were placed proximal and distal to the intended access point. A 24-gauge angiocatheter was used to cannulate the portal vein. Once access was obtained, the sutures were used to secure access and achieve hemostasis. DSA imaging was performed by using a C-arm unit (PowerMobil; Siemens Medical Solutions, Erlangen, Germany) to evaluate flow through the angiocatheter following test injections of $0.1-0.2 \mathrm{~mL}$ of saline and iodinated contrast material $(\mathrm{Om}-$ nipaque 350; GE Healthcare, Princeton, NJ). Next, a 1-mL aliquote of the lipiodol-saline mixture was infused through the angiocatheter with fluoroscopic guidance to confirm intrahepatic delivery and visualize intrahepatic distribution. Prior to MR imaging, rats were euthanized with $2 \mathrm{~mL}$ of Euthasol (Virbac AH, Fort Worth, Tex) to eliminate the necessity for gating and potential for residual motion artifacts during MR imaging.

\section{MR Imaging Studies}

Studies were performed by using a 1.5-T MR imager (Magnetom Avanto; Siemens, Erlangen, Germany) and a 12-channel receiver coil. For all studies, an MGRE pulse sequence was used with the following acquisition parameters: repetition time of 1000 msec, echo time of $2.46 \mathrm{msec}$, and echo train length of 12 , with spacing of $2.7 \mathrm{msec}$; $192 \times 70$ matrix; $240-\mathrm{mm}$ field of view; $75^{\circ}$ flip angle; 3.5-mm section thickness; 900-Hz/pixel bandwidth; and six signals acquired. We reduced effective echo time spacing to $0.9 \mathrm{msec}$ by acquiring a second and third interleaved data set with the echo train delayed such that the echo time for the first echo was $3.36 \mathrm{msec}$, and $4.26 \mathrm{msec}$ for the second and third data sets.

\section{Histologic Evaluation}

Livers were excised and two to three sections were sampled from the targeted hepatic lobe. These specimens were placed in a $-80^{\circ} \mathrm{C}$ freezer overnight. A subset of these specimens were fixed in formaldehyde and embedded in paraffin for histologic examination; Oil-Red-O staining (28) was used to confirm parenchymal delivery of the lipiodol media qualitatively. Histology slides were digitized by using a multichannel automated imaging system (Tissue Gnostics, Vienna, Austria) and assessed (X.Y. and Y.G., each with more than 3 
Figure 1

\section{$\mathrm{CH}_{3} \mathrm{C} \mathrm{H}_{2} \mathrm{OC}(\mathrm{O}) \mathrm{CH}_{2}\left(\mathrm{CH}_{2}\right){ }_{-7} \mathrm{CH}_{2} \mathrm{ClHCH}_{2}\left(\mathrm{CH}_{2}\right)_{-5} \mathrm{CH}$}

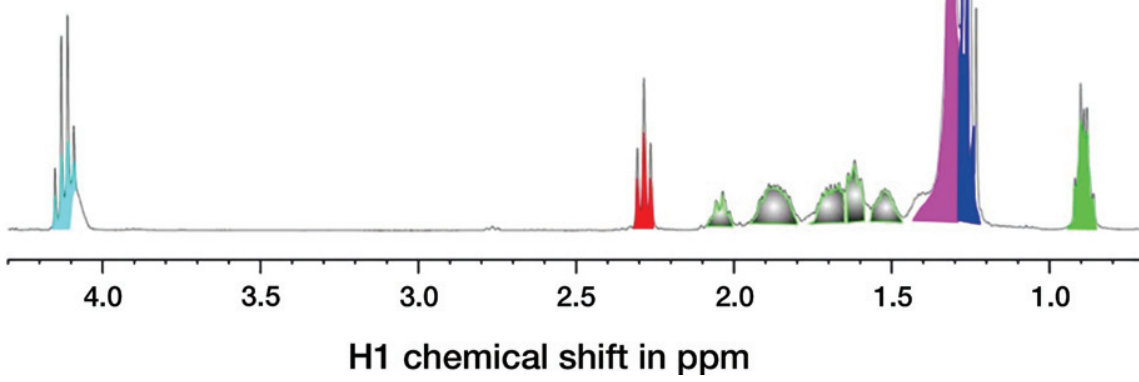

Figure 1: Lipiodol nuclear MR spectrum. ${ }^{1} \mathrm{H}$ spectrum for lipiodol measured (in parts per million) by using 360-MHz nuclear MR spectrometer and approximated chemical structure.

years experience with the assessment of rodent pathology slides).

\section{Data Analysis}

For phantom imaging studies, water $\left(s_{w}\right)$ and lipiodol $\left(s_{L}\right)$ images were calculated by using the previously described VARPRO-based method and either single-peak or multipeak chemical shiftsignal models. An equal relative proton density $(\rho \lambda / M W$, with density $\rho$, molecular weight MW, and $\lambda$ the number of proton per molecule) was assumed for water and lipiodol $\left(\rho_{\mathrm{w}} \lambda_{\mathrm{w}} / \mathrm{MW}_{\mathrm{w}}: \rho_{\mathrm{L}} \lambda_{\mathrm{L}} /\right.$ $\left.\mathrm{MW}_{\mathrm{L}} \approx 1\right)$. With the latter assumptions $(15,21,29)$ one can approximate the lipiodol volume fraction for each voxel based on the corresponding lipiodol signal fraction measurement $\left(s_{L} / s_{W}\right.$ $+s_{L}$ ) (15). Voxelwise lipiodol concentration maps (in grams per milliliter) were thus calculated by means of scaling of the lipiodol volume fraction maps (multiplication by known density of lipiodol $=1.28 \mathrm{~g} / \mathrm{mL}$ ).

A circular 24-mm-diameter region of interest (ROI) was drawn (by X.Y., with more than 5 years experience in fields of signal processing and
MR imaging research) to encompass the lipiodol-infused gel portion of each phantom in the lipiodol concentration maps. Identical ROI sizes were used for each phantom measurement. For the animal model studies, ROIs were drawn to encompass the entire volume of normal liver parenchyma in each section while excluding nonhepatic tissues. These ROIs were drawn for all sections in each animal for complete volumetric three-dimensional liver coverage (liver ROI volumes for these animal model studies ranged from 11.7 to $14.0 \mathrm{~cm}^{3}$ ). A summation of these concentration values and subsequent multiplication of this sum by the voxel size (30) produced a whole-liver lipiodol volume measurement for each animal. For animal model studies, a baseline offset was anticipated (given the presence of endogenous fat within normal liver tissues). This offset was estimated from control animal data sets (those not receiving a lipiodol infusion). Voxelwise subtraction of this offset was performed to produce a second set of corrected whole-liver lipiodol volume measurements for each rat in groups 1,2 , and 3 .

\section{Statistical Analysis}

Pearson correlation coefficients were calculated to compare known lipiodol concentrations (according to phantom composition) to subsequent MR imaging-based lipiodol measurements performed by using either single-peak or multipeak signal models.

Analysis of variance methods were used to evaluate between-group differences for MR imaging-based lipiodol volume measurements (for both singleand multipeak models). Independent $t$ tests were used to compare single- and multipeak lipiodol volume measurements for each dose group. Intraclass correlation was used to evaluate the consistency between MR imagingbased lipiodol volume measurements and the volume of lipiodol administered to each animal via portal vein infusion. These analyses were implemented with a statistical software package (SPSS, Chicago, Ill), with $P$ values less than .05 considered to indicate a statistically significant difference.

\section{Results}

Nuclear MR Spectroscopy and Phantom Studies

The lipiodol ${ }^{1} \mathrm{H}$ nuclear MR spectrum and approximated chemical structure is depicted in Figure 1. A verification of these assignments was performed with ${ }^{13} \mathrm{C}$ attached proton tests, twodimensional ${ }^{1} \mathrm{H}-{ }^{1} \mathrm{H}$ correlation and ${ }^{1} \mathrm{H}-$ ${ }^{13} \mathrm{C}$ heteronuclear multiple-quantum correlation spectroscopy. Lipiodol contains several types of fatty acids as determined by thin layer chromatography and the number of carbon peaks greatly exceeds the number expected from a pure stearate compound. On the basis of these nuclear MR measurements, we estimated the principle high-energy lipiodol spectral peaks $\left(f_{L}\right)$ to be located at $\{-33,-153,-216$, $-242 \mathrm{~Hz}\}$ for subsequent MR imaging studies at $1.5 \mathrm{~T}$ with relative amplitudes $\left(\alpha_{m}\right)$ for these peaks estimated to be $\{0.098,0.082,0.701,0.112\}$, respectively. While five additional peaks were observed in the lipiodol spectrum (grayed zones in spectrum), these were 


\section{Figure 2}

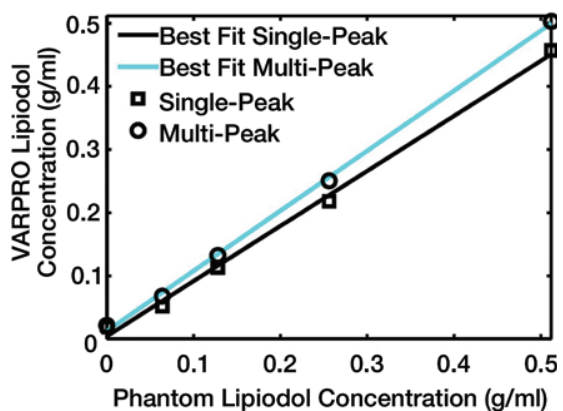

Figure 2: Both single- and multipeak measurements were well correlated to known lipiodol phantom concentration ( $r^{2}>0.99$ for both methods).

of lower amplitude accounting for less than one-third of overall peak area. These five peaks were not specifically assigned and these were not included in our multifrequency signal model during subsequent MR imaging studies.

Both single-peak and multipeak measurements were well correlated to known lipiodol concentration $\left(r^{2}>\right.$ 0.99 for both methods, Fig 2). For control phantom (void of lipiodol content), both single- and multipeak methods overestimated lipiodol concentration; however, for these phantom studies the accuracy of multipeak measurements tended to increase with increasing lipiodol concentration whereas single-peak measurements consistently underestimated lipiodol content with increasing concentration (Table 1).

\section{Animal Model}

During transcatheter infusion procedures, intrahepatic lipiodol delivery in each animal was confirmed with DSA (Fig 3). Lipiodol distribution typically followed one of two potential patterns: either (a) diffuse delivery to multiple lobes or (b) focal delivery to only a limited region. Lipiodol delivery to the lungs via hepatopulmonary communications was not observed in these animals.

Analysis of variance indicated significant differences between intrahepatic lipiodol volume measurements for the four different groups (control animals and animals in groups 1,2 , and 3 , with

\section{Table 1}

\section{Lipiodol Phantom Concentration Measurements}

Phantom Composition

Single-Peak VARPRO

Multipeak VARPRO

Concentration $(\mathrm{mg} / \mathrm{mL})$ Concentration $(\mathrm{mg} / \mathrm{mL})$ Concentration $(\mathrm{mg} / \mathrm{mL})$

0 64

128

256

512

18

51

113

218

457

21

68

132

250

506
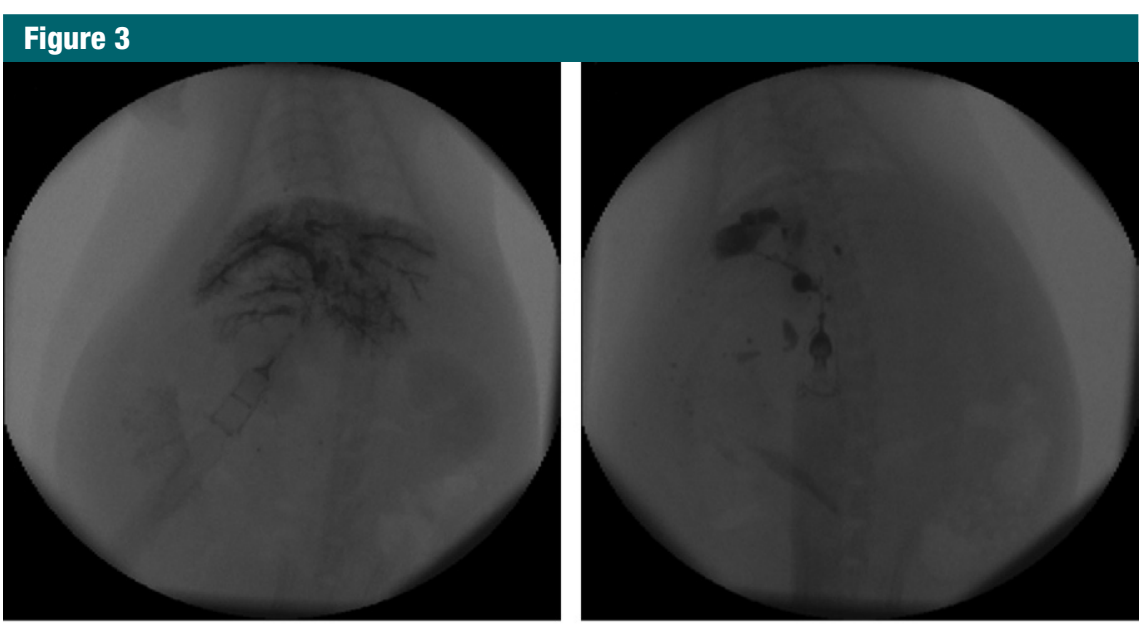

a.

b.

Figure 3: DSA fluoroscopic depiction of the lipiodol distribution patterns in two representative animals: (a) diffuse lipiodol distribution to multiple lobes and (b) focal delivery to only a limited region.

$P \leq .05$ for comparison of single-peak measurements and $P \leq .05$ for comparison of multipeak measurements) (Fig 4). MR imaging-based intrahepatic lipiodol volume measurements significantly increased with increased transcatheter lipiodol dose as indicated by significant linear trends $(P \leq .001)$. For both single- and multipeak approaches, mean lipiodol volume measurements were progressively and significantly higher when comparing between groups receiving different lipiodol doses $(P<.05$ for each comparison). Comparison of MR imaging-based intrahepatic lipiodol volume measurements and the transcatheter-infused dose indicated intraclass correlation coefficients of $0.939(P<.001)$ for single-peak method and $0.933(P<$ .001 ) for the multipeak method (Fig 5). However, as demonstrated in Figure 5 plots, these hepatic MGRE VARPRObased measurements produced lipiodol volume measurements that were offset roughly $0.4 \mathrm{~mL}$ above the injected dose (likely due to the presence of endogenous fat within the rat liver). The calculated mean lipiodol volumes for control animals were roughly $0.39 \mathrm{~mL}$ and $0.46 \mathrm{~mL}$ (offsets for single- and multipeak modeling, respectively). A voxelwise subtraction of this offset produced whole-liver lipiodol volume measurements that more closely agreed with the recorded infusion volumes (Table 2 ). Lipiodol in liver tissue specimens was clearly identified as punctate red droplets within the Oil-Red-O-stained histology slides (Fig 6a); these deposits were not observed in control specimens. For most animals, the lipiodol distribution was also visible during the initial gross 


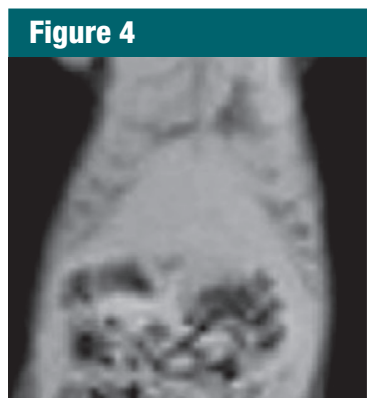

a.

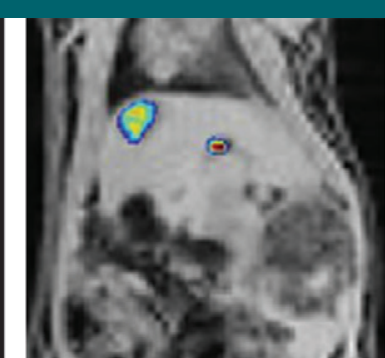

b.

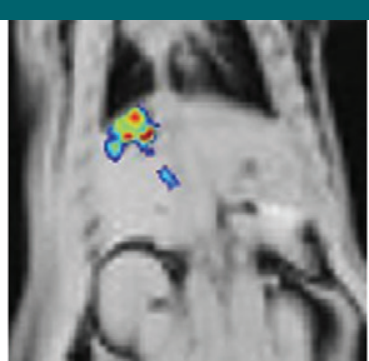

c.

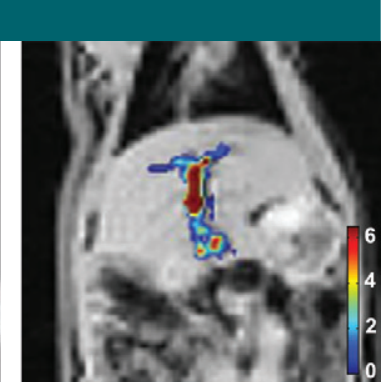

d.

Figure 4: Coronal MGRE MR images with color-coded overlays depict voxelwise estimates of intrahepatic lipiodol concentration (at single central hepatic section position, in grams per milliliter). Representative examples are shown for (a) control animal and treated animals in (b) group 1 (15\% volume lipiodol-saline mixtures), (c) group 2 (30\% volume lipiodol-saline mixtures), and (d) group 3 (50\% volume lipiodolsaline mixtures). Examples shown for treated animals in $\mathbf{b}$ and $\mathbf{c}$ depict focal delivery to a single lobe, whereas in the treated animal shown in $\mathbf{d}$ the lipiodol was largely contained within proximal branches of the portal vein.

\section{Figure 5}

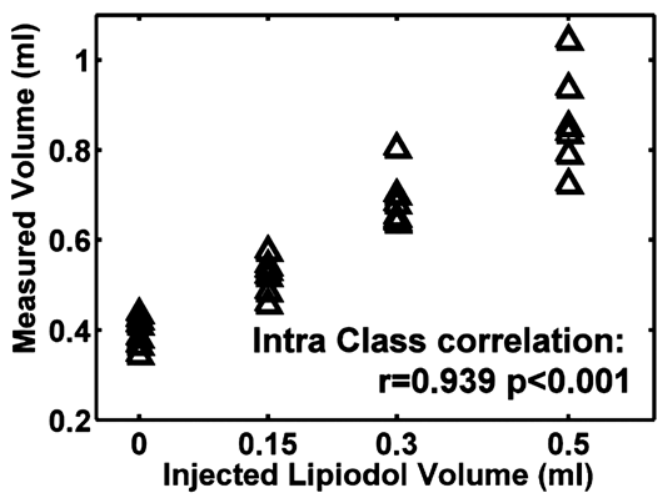

a.

Figure 5: Comparison between MR imaging-based intrahepatic lipiodol volume measurements and the transcatheter-infused dose for control animals and those in groups 1, 2, and 3 by using either (a) single-peak or (b) multipeak VARPRO methods. examination of the excised liver specimens; these focal deposits were depicted as regions of yellow or white discoloration of hepatic tissues that would otherwise be red in color at necropsy (Fig 6b).

\section{Discussion}

These preclinical studies demonstrate the use of chemical shift-based fat-water separation methods for the quantification of lipiodol delivery to the liver. Given the potential benefits of MR imaging over competing modalities, the described methods may have important future implications for monitoring lipiodol-mediated drug delivery during TACE procedures. Therapeutic outcomes are anticipated to be strongly correlated to the volume of drug-lipiodol cocktail delivered to liver tumor tissues. Quantitative measurements of intrahepatic lipiodol distribution may ultimately permit early prediction of treatment response.

Both single-peak and multipeak modeling approaches produced lipiodol measurements strongly correlated to phantom composition. Both approaches overestimated lipiodol concentration for the control phantom but produced measurements that were more accurate with increasing lipiodol concentration.
These findings are consistent with prior studies using chemical shift methods for hepatic fat quantification; for these prior studies, the overestimation of fat was attributed to low fat signal content and associated noise bias (31).

For animal model studies, both single-peak and multipeak approaches produced lipiodol measurements that were strongly correlated to the infused lipiodol dose. However, while strongly correlated, both approaches produced measurements that included a consistent baseline offset proportional to the lipiodol volume estimated for the control animals. This finding was not entirely unexpected given the similar position 


\section{Table 2}

\begin{tabular}{llll}
\multicolumn{2}{l}{ Hepatic Lipiodol Volume Measurements } \\
Group & Infused Lipiodol Dose $(\mathrm{mL})$ & $\begin{array}{l}\text { Single-Peak VARPRO } \\
\text { Measurement }(\mathrm{mL})\end{array}$ & $\begin{array}{l}\text { Multipeak VARPRO } \\
\text { Measurement }(\mathrm{mL})\end{array}$ \\
\hline Control & 0 & $0.39 \pm 0.04$ & $0.46 \pm 0.04$ \\
\hline 1 & 0.15 & $0.52 \pm 0.03 / 0.13 \pm 0.03^{\star}$ & $0.64 \pm 0.04 / 0.18 \pm 0.04^{\star}$ \\
2 & 0.30 & $0.68 \pm 0.06 / 0.29 \pm 0.06^{\star}$ & $0.81 \pm 0.05 / 0.26 \pm 0.05^{\star}$ \\
3 & 0.50 & $0.86 \pm 0.11 / 0.48 \pm 0.11^{*}$ & $1.030 .13 / 0.57 \pm 0.13^{\star}$ \\
\hline
\end{tabular}

Note.-For VARPR0 measurements, data are mean \pm standard deviation.

* Additional set of measurements that have been adjusted for baseline offset owing to endogenous hepatic fat (voxelwise subtraction of corresponding control measurements).

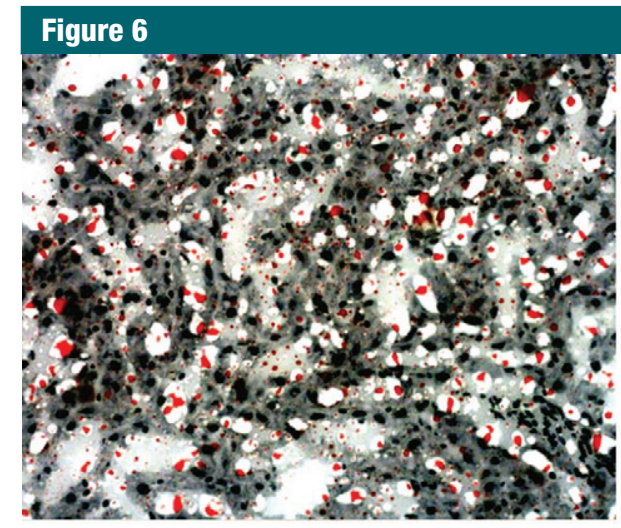

a.

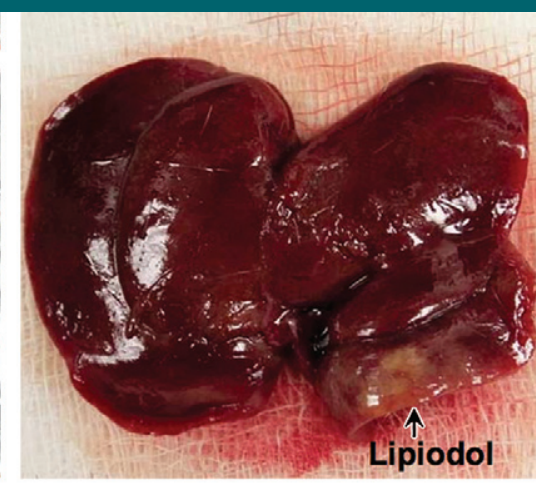

b.

Figure 6: (a) Oil-Red-0 staining of sectioned liver samples and (b) representative liver specimen.

(a) Lipiodol in liver tissue specimens were identified as red droplets in the Oil-Red-0 histology slides.

(b) Focal lipiodol deposits were visible in excised liver specimen depicted as regions of yellow or white discoloration of hepatic tissues

of the spectral peaks for lipiodol and hepatic fat tissues (21); the calculated mean lipiodol volumes for control animals corresponded to fat fractions of $3 \%-4 \%$, both well within ranges previously reported for normal liver parenchyma $(32,33)$. Voxelwise subtraction of this offset concentration produced whole-liver lipiodol measurements that were consistent with the bulk volumes infused. While effective for correcting lipiodol measurement offsets in normal liver tissues, the latter approach may be ineffective in diseased hepatic tissues or for measurements within tumor tissues (34). Accurate measurements in the latter settings may require baseline chemical shift fat measurements prior to TACE procedures to allow for patientspecific offset corrections. CT lipiodol imaging approaches are reliant only on the $x$-ray attenuation resulting from the iodine component of the lipiodol medium, thus, the accuracy of CT methods is not adversely affected by variations in endogenous hepatic fat content.

For phantom studies, the singlepeak approach underestimated lipiodol concentrations. For each phantom, the MR imaging-based approximation of lipiodol concentration was higher when using the multipeak method compared with corresponding measurement performed by using the single-peak method. Similarly, for subsequent animal model studies, the multipeak approach yielded larger lipiodol volume measurements for each group. Both methods produced measurements strongly correlated to the infused dose (intraclass correlations of 0.939 and 0.933 for single- and multipeak methods, respectively). However, at subtraction of the baseline offset (estimated from control group), the single-peak measurements tended to more accurately reflect the infused lipiodol dose (smaller absolute differences between infused lipiodol volume and offset-corrected MR imaging-based measurement in each animal; Table 2); additional studies are necessary to validate these findings and rigorously examine the discrepancy between these signal models for phantom versus intrahepatic measurements. Inherent differences in signal-to-noise ratio and tissue relaxation characteristics between phantom and animal settings may have contributed to the latter findings. Of importance, a model-based correction for $\mathrm{T} 2 *$ relaxation was not performed in our study; these corrections can improve quantitative accuracy during multiecho chemical shift-based fat-water measurements $(24,27,35)$ and thus may also lead to improved accuracy during intrahepatic lipiodol measurements. Further investigation of signal models that include both fat and water relaxation rates or involve separate modeling of endogenous fat and lipiodol signal components (rather than the subtraction of an estimated baseline) may serve to improve the accuracy of both single- and multipeak methods. However, the efficacy of the latter approach would presuppose a sufficient difference between lipiodol and endogenous fat proton spectra; a rigorous definition of these spectral differences could be a valuable focus of future investigations beyond our current feasibility studies. The practical implication of an inability to discriminate endogenous fat from lipiodol is unclear. Primary liver tumors (HCCs) are the primary indication for TACE. HCCs rarely contain fat (36). Fat content can be observed in some cases of early stage HCC (1.0-1.5 cm) (37) but these are often treated with resection or percutaneous ablation rather than TACE. Fat deposition in larger, well-differentiated HCC is uncommon (38).

The standard deviation of MR imaging-based lipiodol volume measurements 
was markedly larger for group 3 animals. This larger spread in the measured lipiodol volumes (compared with that observed for control animals and those in groups 1 and 2) may have been the result of variations in the distribution volume between animals included in this group. For group 3 rats, the infused saline-lipiodol mixture was relatively viscous; it is plausible that this increased viscosity (a) limited the distal distribution of the infused volume (with a small volume of lipiodol remaining within the proximal portal vein) or (b) resulted in retrograde reflux of a portion of the infused volume into the mesentery. Region of interest measurements included only hepatic parenchyma and the distal branches of the portal vein embedded within the liver volume.

Our study had several limitations. First, these studies were performed with lipiodol delivered to normal liver parenchyma without a liver tumor present and with lipiodol infused through a catheter positioned within the portal vein rather than in a hepatic artery (per conventional TACE). The portal vein was chosen given its larger diameter and surgical accessibility. Given the promising outcomes of these initial feasibility studies, future investigations are now warranted to evaluate the efficacy of these methods for the quantification of lipiodol delivery to liver tumors (39); such investigations should also compare the proposed MR imaging-based lipiodol distribution measurements to longitudinal outcomes, but this was beyond the scope of the current validation study. Finally, ex vivo imaging permitted long repetition times with a relatively high flip angle $\left(75^{\circ}\right)$ for superior signal-to-noise ratio; gating procedures and appropriately optimized sequence parameters will be necessary to avoid respiratory motion artifacts and potential T1 bias $(31,40,41)$ during future in vivo imaging studies.

Practical application: We have demonstrated that chemical shift-based MR imaging methods can be used to accurately quantify transcatheter lipiodol delivery to liver tissues. Lipiodol serves a critical role as both drug carrier and viscous embolic agent during TACE procedures. Chemical shift-based MR imaging measurements may ultimately serve as a radiologic means to noninvasively estimate lipiodol-mediated drug delivery and tissue embolization.

Acknowledgments: We sincerely thank Scott Reeder, $\mathrm{MD}, \mathrm{PhD}$, for his advice and invaluable comments during the preparation of the manuscript and Catherine D. G. Hines, PhD, for instruction regarding optimal preparation of fatwater MR imaging phantoms.

Disclosures of Potential Conflicts of Interest: X.Y. No potential conflicts of interest to disclose. Y.G. No potential conflicts of interest to disclose. W.L. No potential conflicts of interest to disclose. E.H. No potential conflicts of interest to disclose. Z.Z. No potential conflicts of interest to disclose. J.N. No potential conflicts of interest to disclose. R.A.K. No potential conflicts of interest to disclose. D.H. No potential conflicts of interest to disclose. A.K.K. No potential conflicts of interest to disclose. R.A.O. No potential conflicts of interest to disclose. A.C.L. No potential conflicts of interest to disclose.

\section{References}

1. Yamada R, Sato M, Kawabata M, Nakatsuka H, Nakamura K, Takashima S. Hepatic artery embolization in 120 patients with unresectable hepatoma. Radiology 1983;148(2):397-401.

2. Liapi E, Georgiades CC, Hong K, Geschwind JF. Transcatheter arterial chemoembolization: current technique and future promise. Tech Vasc Interv Radiol 2007;10(1):2-11.

3. Lo CM, Ngan H, Tso WK, et al. Randomized controlled trial of transarterial lipiodol chemoembolization for unresectable hepatocellular carcinoma. Hepatology 2002;35(5): 1164-1171.

4. Yamada R, Nakatsuka H, Nakamura K, et al. Hepatic artery embolization in 32 patients with unresectable hepatoma. Osaka City Med J 1980;26(2):81-96.

5. Liapi E, Geschwind JF. Chemoembolization for primary and metastatic liver cancer. Cancer J 2010;16(2):156-162.

6. Rizvi S, Camci C, Yong Y, et al. Is post-Lipiodol CT better than i.v. contrast CT scan for early detection of HCC? A single liver transplant center experience. Transplant Proc 2006; 38(9):2993-2995.

7. Choti MA, Geschwind JF. Preoperative sequential TACE and PVE to increase resectability in the cirrhotic patient with HCC. Gastrointest Cancer Res 2008;2(1): $47-48$.

8. Matsumoto K, Nojiri J, Takase Y, et al. Cerebral lipiodol embolism: a complication of transcatheter arterial chemoembolization for hepatocellular carcinoma. Cardiovasc Intervent Radiol 2007;30(3):512-514.

9. Gusani NJ, Balaa FK, Steel JL, et al. Treatment of unresectable cholangiocarcinoma with gemcitabine-based transcatheter arterial chemoembolization (TACE): a singleinstitution experience. J Gastrointest Surg 2008;12(1):129-137.

10. Taguchi K, Funama Y, Zhang M, Fishman EK, Geschwind JF. Quantitative measurement of iodine concentration in the liver using abdominal C-arm computed tomography. Acad Radiol 2009;16(2):200-208.

11. Wang D, Jin B, Lewandowski RJ, et al. Quantitative 4D transcatheter intraarterial perfusion MRI for monitoring chemoembolization of hepatocellular carcinoma. J Magn Reson Imaging 2010;31(5):1106-1116.

12. Guo Y, Zhang Y, Jin N, et al. Electroporation-mediated transcatheter arterial chemoembolization in the rabbit VX2 liver tumor model. Invest Radiol 2012;47(2):116-120.

13. Park JK, Rhee TK, Cashen TA, et al. MR imaging assessment of changes in renal function with renal artery stent placement in swine. J Vasc Interv Radiol 2007;18(11):1409-1416.

14. De Santis M, Alborino S, Tartoni PL, Torricelli P, Casolo A, Romagnoli R. Effects of lipiodol retention on MRI signal intensity from hepatocellular carcinoma and surrounding liver treated by chemoembolization. Eur Radiol 1997;7(1):10-16.

15. Reeder SB, Hines CD, Yu H, McKenzies CA, Brittains JH. On the definition of fat-fraction for in vivo fat quantification with magnetic resonance imaging [abstr]. In: Proceedings of the Seventeenth Meeting of the International Society for Magnetic Resonance in Medicine. Berkeley, Calif: International Society for Magnetic Resonance in Medicine, 2009; 211.

16. Dixon WT. Simple proton spectroscopic imaging. Radiology 1984;153(1):189-194.

17. Glover GH. Multipoint Dixon technique for water and fat proton and susceptibility imaging. J Magn Reson Imaging 1991;1(5):521530 .

18. Reeder SB, Wen Z, Yu H, et al. Multicoil Dixon chemical species separation with an iterative least-squares estimation method. Magn Reson Med 2004;51(1):35-45.

19. Reeder SB, Brittain JH, Grist TM, Yen YF Least-squares chemical shift separation for (13)C metabolic imaging. J Magn Reson Imaging 2007;26(4):1145-1152.

20. Yokoo T, Bydder M, Hamilton G, et al. Nonalcoholic fatty liver disease: diagnostic and 
fat-grading accuracy of low-flip-angle multiecho gradient-recalled-echo MR imaging at 1.5 T. Radiology 2009;251(1):67-76.

21. Bultman EM, Hines CD, Block WF, Reeder SB. MR validation and quantification of TACE treatment through direct visualization of ethiodol with chemical shift based water-fat imaging [abstr]. In: Proceedings of the Seventeenth Meeting of the International Society for Magnetic Resonance in Medicine. Berkeley, Calif: International Society for Magnetic Resonance in Medicine, 2009; 1749.

22. Hernando D, Haldar JP, Sutton BP, Ma J, Kellman P, Liang ZP. Joint estimation of water/fat images and field inhomogeneity map. Magn Reson Med 2008;59(3): 571-580.

23. Hernando D, Kellman P, Haldar JP, Liang ZP. Robust water/fat separation in the presence of large field inhomogeneities using a graph cut algorithm. Magn Reson Med 2010;63(1):79-90.

24. Yu H, Shimakawa A, McKenzie CA, Brodsky E, Brittain JH, Reeder SB. Multiecho water-fat separation and simultaneous R2* estimation with multifrequency fat spectrum modeling. Magn Reson Med 2008; 60(5):1122-1134.

25. Golub GH, Pereyra V. The differentiation of pseudoinverses and nonlinear least squares problems whose variables separate. SIAM J Numer Anal 1973;10(2):413-432.

26. Hines CD, Yu H, Shimakawa A, McKenzie CA, Brittain JH, Reeder SB. T1 independent, $\mathrm{T}^{*}$ corrected MRI with accurate spectral modeling for quantification of fat: validation in a fat-water-SPIO phantom. J Magn Reson Imaging 2009;30(5):1215-1222.
27. Hernando D, Liang ZP, Kellman P. Chemical shift-based water/fat separation: a comparison of signal models. Magn Reson Med 2010;64(3):811-822.

28. Wang HN, Wang YR, Liu GQ, et al. Inhibition of hepatic interleukin-18 production by rosiglitazone in a rat model of nonalcoholic fatty liver disease. World J Gastroenterol 2008;14(47):7240-7246.

29. Reeder SB, Hines CD, Yu H, McKenzies CA, Brittains JH. Relationship between proton-density fat-fraction and true fat concentration for in vivo fat quantification with magnetic resonance imaging [abstr]. In: Proceedings of the Nineteenth Meeting of the International Society for Magnetic Resonance in Medicine. Berkeley, Calif: International Society for Magnetic Resonance in Medicine, 2011; 805.

30. Klausmann F, Ludwig U, Honal M, Konig D, Deibert P, Huff S. Accuracy of wholebody fat quantification with MRI: a comparison to air-displacement plethysmography [abstr]. In: Proceedings of the Eighteenth Meeting of the International Society for Magnetic Resonance in Medicine. Berkeley, Calif: International Society for Magnetic Resonance in Medicine, 2010; 57.

31. Liu CY, McKenzie CA, Yu H, Brittain JH, Reeder SB. Fat quantification with IDEAL gradient echo imaging: correction of bias from $\left.\mathrm{T}_{1}\right)$ and noise. Magn Reson Med 2007; 58(2):354-364.

32. Sijens PE, Edens MA, Bakker SJ, Stolk RP. MRI-determined fat content of human liver, pancreas and kidney. World J Gastroenterol 2010;16(16):1993-1998.

33. O'Regan DP, Callaghan MF, WylezinskaArridge M, et al. Liver fat content and $\mathrm{T} 2 *$ : simultaneous measurement by using breath- hold multiecho MR imaging at $3.0 \mathrm{~T}-$ feasibility. Radiology 2008;247(2):550-557.

34. Ma X, Holalkere NS, Kambadakone R A, Mino-Kenudson M, Hahn PF, Sahani DV. Imaging-based quantification of hepatic fat: methods and clinical applications. RadioGraphics 2009;29(5):1253-1277.

35. Yu H, McKenzie CA, Shimakawa A, et al. Multiecho reconstruction for simultaneous water-fat decomposition and $\mathrm{T} 2 *$ estimation. J Magn Reson Imaging 2007;26(4): 1153-1161.

36. Balci NC, Befeler AS, Bieneman BK, Fattahi R, Saglam S, Havlioglu N. Fat containing HCC: findings on CT and MRI including serial contrast-enhanced imaging. Acad Radiol 2009;16(8):963-968.

37. Kutami R, Nakashima Y, Nakashima O, Shiota K, Kojiro M. Pathomorphologic study on the mechanism of fatty change in small hepatocellular carcinoma of humans. J Hepatol 2000;33(2):282-289.

38. Prasad SR, Wang H, Rosas H, et al. Fat containing lesions of the liver: radiologicpathologic correlation. RadioGraphics 2005; 25(2):321-331.

39. Guo Y, Zhang Y, Klein R, et al. Irreversible electroporation therapy in the liver: longitudinal efficacy studies in a rat model of hepatocellular carcinoma. Cancer Res 2010;70(4):1555-1563.

40. Bydder M, Yokoo T, Hamilton G, et al. Relaxation effects in the quantification of fat using gradient echo imaging. Magn Reson Imaging 2008;26(3):347-359.

41. Jin N, Zhang Z, Zhang L, Lu G, Larson AC. Respiratory self-gated multiple gradient recalled echo sequence for free-breathing abdominal R2* mapping. Magn Reson Med 2011;66(1):207-212. 